\title{
CRESCIMENTO DE MUDAS DE Eucalyptus grandis UTILIZANDO LODO DE ESGOTO, FIBRA DE COCO E PALHA DE CAFÉ IN NATURA
}

\author{
Marcos Vinicius Winckler Caldeira ${ }^{1}$, Elzimar de Oliveira Gonçalves ${ }^{1}$, Paulo André Trazzi ${ }^{2}$, William \\ Macedo Delarmelina ${ }^{3}$, Rafael Luiz Frinhani Rocha ${ }^{4}$ \\ ${ }^{1}$ Eng. Florestal, Dr., Depto. de Ciências Florestais e da Madeira, UFES, Jerônimo Monteiro, ES, Brasil - mvwcaldeira@gmail.com; \\ elzimarog@yahoo.com.br \\ ${ }^{2}$ Eng. Florestal, M.Sc., Doutorando em Engenharia Florestal, UFPR, Curitiba, PR, Brasil - patrazzi@hotmail.com \\ ${ }^{3}$ Eng. Florestal, Mestrando em Ciências Florestais, UFES, Jerônimo Monteiro, ES, Brasil - williamdm@hotmail.com \\ ${ }^{4}$ Acadêmico do Instituto Federal de Educação, Ciência e Tecnologia do Espírito Santos (IFES), Campus de Alegre, ES, Brasil - \\ rafaelfrinhanii@gmail.com
}

Recebido para publicação: 10/02/2012 - Aceito para publicação: 10/12/2013

\begin{abstract}
Resumo
O presente estudo teve como objetivo testar a utilização de lodo de esgoto e resíduos orgânicos na composição de substratos para a produção de mudas de Eucalyptus grandis Hill ex Maiden. O experimento foi conduzido no Viveiro Florestal do Centro de Ciências Agrárias da UFES, em Alegre, ES, constituindo-se de delineamento inteiramente casualizado, com seis repetições e 10 tratamentos: $100 \%$ LE, 80\% LE + 20\% FC, 60\% LE + 40\% FC, 40\% LE + 60\% FC, 20\% LE + 80\% FC, 80\% LE + $20 \%$ PCN, 60\% LE + 40\% PCN, 40\% LE + 60\% PCN, 20\% LE + 80\% PCN, e uma testemunha 100\% SC. Foram realizadas análises químicas e físicas dos tratamentos e aos 90 dias após a semeadura determinaram-se as características morfológicas das plantas. Os resultados indicaram que a densidade aparente, a porosidade total, a macro e microporosidade, o pH, a disponibilidade de água, K, P, Ca, Mg e sais solúveis foram influenciados pela maior ou menor proporção de lodo de esgoto, fibra de coco ou palha de café in natura. O tratamento $80 \%$ LE + 20\% PCN apresentou os maiores valores de altura, diâmetro do coleto, massa seca da parte aérea, massa seca radicular e massa seca total.

Palavras-chave: Biossólido; fibras vegetais; eucalipto; características morfológicas.
\end{abstract}

\begin{abstract}
Growth of seedlings of Eucalyptus grandis using sewage sludge, coconut fiber and straw of coffee in natura. The present study aimed to test the use of sewage sludge and organic waste in substrate composition for the production of seedlings of Eucalyptus grandis Hill ex Maiden. The experiment was conducted at the Forest Nursery Center for Agricultural Sciences UFES in Alegre, ES. It was developed in completely randomized design with six replications and 10 treatments $100 \% \mathrm{LE}, 80 \% \mathrm{LE}+20 \% \mathrm{FC}$, $60 \% \mathrm{LE}+40 \% \mathrm{FC}, 40 \% \mathrm{LE}+60 \% \mathrm{FC}, 20 \% \mathrm{LE}+80 \% \mathrm{FC}, 80 \% \mathrm{LE}+20 \% \mathrm{PCN}, 60 \% \mathrm{LE}+40 \%$ PCN, $40 \%$ LE $+60 \%$ PCN, $20 \%$ LE $+80 \%$ PCN, and a witness $100 \%$ SC. Analyses of chemical and physical treatments, and at 90 days after sowing we determined the morphological characteristics of the plants. The results indicated that bulk density, total porosity, macro and microporosity, $\mathrm{pH}$, availability of water, K, P, Ca, Mg, and soluble salts were influenced by a greater or lesser proportion of sewage sludge, coir straw or fresh coffee. Treatment $80 \%$ LE $+20 \%$ PCN presented the highest values of height, stem diameter, shoot dry mass, root dry mass and total dry mass.

Keywords: Biosolids; plant fibers; eucalyptus; morphological characteristics.
\end{abstract}

\section{INTRODUÇÃO}

A adoção de padrões técnicos e procedimentos adequados na composição dos substratos poderão melhorar a qualidade das mudas produzidas, independentemente da finalidade a que sejam destinadas, seja para a composição de povoamentos comerciais, seja para a recuperação de áreas degradadas.

Um dos fatores que determinam o padrão de qualidade das mudas é o substrato, meio no qual as plantas fixarão suas raízes, devendo ser capaz de reter líquidos e disponibilizar nutrientes às plantas (MELLO et al., 2006). Como a diversidade de opções de materiais a serem utilizados é grande, não há um 
substrato perfeito para todas as condições e espécies. É sempre preferível usar componente de um substrato em forma de mistura, visto que os mesmos apresentam características desejáveis e indesejáveis à planta quando usados isoladamente (WENDLING; GATTO, 2002). Por essa razão, tem crescido a utilização de diversos resíduos na produção de mudas florestais, dando continuidade a um processo evolutivo que se iniciou com a substituição gradativa da terra de subsolo por materiais orgânicos. De acordo com Neves et al. (2010), essa prática tem conferido um caráter cada vez mais sustentável às atividades de produção de mudas, minimizando o impacto ambiental que seria provocado com a disposição inadequada desses resíduos na natureza.

Nesse sentido, o lodo de esgoto, um subproduto do tratamento das águas residuais das estações de tratamento com características biológicas, físicas e químicas desejáveis para sua utilização em áreas agrícolas (MORAES NETO et al., 2007), surge como uma alternativa viável de utilização para o crescimento inicial das mudas, pelo fato de ser considerado um excelente fornecedor de matéria orgânica e, portanto, capaz de melhorar as propriedades físicas do solo (JORGE et al., 1991). A composição de substratos por meio do uso do lodo de esgoto, que é rico em fósforo e nitrogênio, além de outros nutrientes encontrados em menores concentrações, representa uma alternativa para a sua destinação final, refletindo em economia de fertilizantes e benefícios ambientais (TRIGUEIRO; GUERRINI, 2003). A utilização de lodo de esgoto como substrato para a produção de mudas pode contribuir para minimizar a contaminação ambiental por elementos potencialmente tóxicos (ROCHA et al., 2013).

O uso da fibra de coco é uma alternativa para a produção de substratos, sendo de fácil obtenção e baixo custo, nas regiões produtoras, por ser um resíduo da exploração comercial da água de coco. A sua composição química é dependente de variações, como época do ano, fonte e quantidade de chuva. A fibra de coco (Cocos nucifera L.) possui excelentes qualidades físicas e químicas quando utilizada como substrato, tais como alta retenção de umidade, resistência à degradação e uniformidade, além de ser livre de patógenos e de ervas daninhas. Devido às suas propriedades de retenção de água, aeração do meio de cultivo e de estimulador do enraizamento, a fibra de coco é um excelente material orgânico para formulações de substratos. O uso dessa fibra pode reduzir substancialmente a necessidade de irrigações ao longo do dia, principalmente no inverno, quando a taxa de transpiração é menor (NUNES, 2000; KÄMPF, 2005; FERRARI, 2003).

A cafeicultura dá origem a um volume elevado de resíduos, principalmente a palha de café, cuja utilização tem sido objeto de diversos estudos, visto que ela deve possuir um destino adequado (VEGRO; CARVALHO, 1994), como na composição de substratos. Matos et al. (1998) encontraram, para cascas de frutos de cafeeiro processados por via seca, valores, em dag. $\mathrm{kg}^{-1}$ de matéria seca, de 1,47 de $\mathrm{N}$ total, 0,17 de fósforo total, 0,81 de cálcio, 3,66 de potássio, $30 \mathrm{mg} \cdot \mathrm{kg}^{-1}$ de zinco e $25 \mathrm{mg} \cdot \mathrm{kg}^{-1}$ de cobre.

Nesse sentido, o objetivo deste trabalho foi testar a utilização de lodo de esgoto e resíduos orgânicos em diferentes substratos e sua recomendação para a produção de mudas de Eucalyptus grandis Hill ex Maiden.

\section{MATERIAL E MÉTODOS}

O experimento foi realizado no Viveiro Florestal do Departamento de Engenharia Florestal (DEF) do Centro de Ciências Agrárias (CCA) da UFES, no município de Alegre, ES, localizado na latitude 204' S e longitude 413' $\mathrm{W}$, com altitude média de $120 \mathrm{~m}$. O clima enquadra-se no tipo Cwa (inverno seco e verão chuvoso), de acordo com a classificação de Köppen, sendo a temperatura média anual de $24,1^{\circ} \mathrm{C}$, com máximas diárias de $31^{\circ} \mathrm{C}$ e mínimas de $20,2^{\circ} \mathrm{C}$ e precipitação anual média de $1.104 \mathrm{~mm}$.

O lodo de esgoto (LE) foi doado pela empresa Foz do Brasil S.A., sendo proveniente da Estação de Tratamento de Esgoto da empresa, localizada no município de Cachoeiro de Itapemirim, ES. As quantidades de metais pesados presentes nesse material (Tabela 1) foram determinadas por meio de análise química fornecida pela própria empresa, que doou o material, para verificar se o mesmo encontra-se dentro dos limites estipulados pela Resolução no 375/2006 do Conselho Nacional do Meio Ambiente (CONAMA, 2006). O material ficou exposto a pleno sol em ambiente aberto por 30 dias e foi posteriormente passado por uma peneira de aço com malha de $2 \mathrm{~mm}$, para homogeneização das partículas.

A fibra de coco (FC) e o substrato comercial (SC) foram provenientes da empresa Fibria Celulose S.A., Unidade Aracruz, ES. A palha de café in natura (PCN) foi adquirida de doações de produtores agrícolas do município de Muniz Freire, ES. 
Tabela 1. Análise química do lodo de esgoto proveniente da Estação de Tratamento de Esgoto Foz do Brasil, de Cachoeiro de Itapemirim, ES.

Table 1. Chemical analysis of sewage sludge from the Wastewater Treatment Station of Foz Brazil, Cachoeiro de Itapemirim, ES.

\begin{tabular}{lcc}
\hline Parâmetros & Resultados analíticos & CONAMA 375/2006 \\
\hline Arsênio & $*<0,5 \mathrm{mg} \cdot \mathrm{dm}^{-3}$ & $41 \mathrm{mg} \cdot \mathrm{kg}^{-1}$ \\
Bário & $156 \mathrm{mg} \cdot \mathrm{dm}^{-3}$ & $1300 \mathrm{mg} \cdot \mathrm{kg}^{-1}$ \\
Cádmio & $<0,053 \mathrm{mg} \cdot \mathrm{dm}^{-3}$ & $39 \mathrm{mg} \cdot \mathrm{kg}^{-1}$ \\
Chumbo & $29 \mathrm{mg} \cdot \mathrm{dm}^{-3}$ & $300 \mathrm{mg} \cdot \mathrm{kg}^{-1}$ \\
Cobre & $98 \mathrm{mg} \cdot \mathrm{dm}^{-3}$ & $1500 \mathrm{mg} \cdot \mathrm{kg}^{-1}$ \\
Cromo & $26 \mathrm{mg} \cdot \mathrm{dm}^{-3}$ & $1000 \mathrm{mg} \cdot \mathrm{kg}^{-1}$ \\
Molibdênio & $3,5 \mathrm{mg} \cdot \mathrm{dm}^{-3}$ & $50 \mathrm{mg} \cdot \mathrm{kg}^{-1}$ \\
Níquel & $11 \mathrm{mg} \cdot \mathrm{dm}^{-3}$ & $420 \mathrm{mg} \cdot \mathrm{kg}^{-1}$ \\
Selênio & $<0,5 \mathrm{mg}^{-3} \mathrm{dm}^{-3}$ & $100 \mathrm{mg} \cdot \mathrm{kg}^{-1}$ \\
Zinco & $409 \mathrm{mg} \cdot \mathrm{dm}^{-3}$ & $2800 \mathrm{mg} \cdot \mathrm{kg}^{-1}$ \\
Fósforo total & $4128 \mathrm{mg} \cdot \mathrm{dm}^{-3}$ & - \\
pH (suspensão a 5\%) & $5,2 \mathrm{mg} \cdot \mathrm{dm}^{-3}$ & - \\
Enxofre & $1,3 \%$ & - \\
Nitrogênio total Kjeldahl & $5646 \mathrm{mg} \cdot \mathrm{dm}^{-3}$ & - \\
Nitrogênio amoniacal & $60 \mathrm{mg} \cdot \mathrm{dm}^{-3}$ & - \\
Carbono orgânico total & $16 \%$ & - \\
Potássio & $1623 \mathrm{mg} \cdot \mathrm{dm}^{-3}$ & - \\
Sódio & $399 \mathrm{mg} \cdot \mathrm{dm}^{-3}$ & - \\
\hline
\end{tabular}

*Valores em base seca.

Os tratamentos foram obtidos misturando-se o lodo de esgoto com os resíduos vegetais (fibra de coco e palha de café in natura), com base no volume dos resíduos nas proporções de 100:0, 80:20, 60:40, 40:60 e 20:80 (lodo de esgoto:resíduo vegetal), e uma testemunha, constituída por substrato comercial, totalizando 10 tratamentos.

Para a produção das mudas, foram utilizados tubetes cilindro-cônicos com capacidade de $280 \mathrm{~cm}^{3}$, objetivando oferecer maior espaço disponível para o desenvolvimento radicular das mudas, tendo em vista a granulometria grosseira dos materiais testados.

No momento da mistura dos materiais, foram adicionadas as quantidades de fertilizantes conforme recomendação proposta por Gonçalves et al. (2000), para o Eucalyptus. Não foram realizadas adubações de cobertura, a fim de avaliar o potencial das misturas dos substratos com sua ausência. Para a produção de mudas em tubetes de polipropileno, utilizaram-se $750 \mathrm{~g}$ de sulfato de amônio, $1667 \mathrm{~g}$ de superfosfato simples e $100 \mathrm{~g}$ de cloreto de potássio por metro cúbico de substrato. Optou-se por utilizar fertilizantes em pó, pela facilidade de homogeneização no substrato. Antes da adubação, foram separadas amostras de cada tratamento, para a posterior realização das análises químicas e físicas.

Após a realização da semeadura manual direta nos tubetes, quando as plântulas alcançaram dois ou três pares de folhas, realizou-se o raleio, que consistiu na eliminação das demais mudas, deixando apenas uma por tubete.

O presente estudo foi montado em um delineamento inteiramente casualizado (DIC), com dez tratamentos e seis repetições, sendo que para cada repetição foi considerada a média de quatro mudas escolhidas aleatoriamente de um total de 24 mudas por tratamento. Cada tratamento foi acondicionado em bandejas, com capacidade de 54 células, em bancadas suspensas a 80 centímetros de altura do solo, na casa de sombra, coberta com tela que permite a passagem de $50 \%$ da luminosidade e irrigadas quatro vezes ao dia, por sistema de irrigação automático em uma lâmina diária de $12 \mathrm{~mm}$, uma média de $3 \mathrm{~mm}$ por irrigação.

Após 60 dias da semeadura, as mudas foram levadas para a área de rustificação, onde ficaram expostas a pleno sol por 30 dias, sob sistema de irrigação automático, duas irrigações no período da manhã e duas à tarde, em um total de $12 \mathrm{~mm}$ diários. Aos 90 dias após a semeadura, foram determinadas as características morfológicas de todas as plantas, sendo analisadas as seguintes variáveis: altura da parte aérea, diâmetro do coleto, massa seca da parte aérea, massa seca radicular e massa seca total. 
Para a análise química dos substratos, utilizou-se o método da EMBRAPA (SILVA et al., 2009) para a determinação dos teores disponíveis de macronutrientes, $\mathrm{pH}$, alumínio trocável (Al), acidez trocável $(\mathrm{H}+\mathrm{Al})$, capacidade de troca de cátions a pH 7 (CTC), soma de bases (SB), saturação por bases (V) e saturação por alumínio (m) nos substratos utilizados.

A caracterização física dos substratos foi realizada no Laboratório de Análises de Substratos para Plantas do Departamento de Horticultura e Silvicultura da Universidade Federal do Rio Grande do Sul (UFRGS), conforme metodologia constante na Instrução Normativa ${ }^{\circ} 17$ do Ministério da Agricultura, Pecuária e Abastecimento (MAPA, 2007).

Os dados referentes às características morfológicas foram submetidos a análise estatística, e para comparação de médias de tratamentos utilizou-se o teste Scott-Knott, a nível de 5\% de significância, por meio da utilização do programa computacional SAEG ${ }^{\circledR}$.

\section{RESULTADOS E DISCUSSÃO}

\section{Características físicas dos substratos}

Houve uma tendência de aumento da densidade dos substratos à medida que foram adicionadas maiores proporções de lodo de esgoto aos componentes fibra de coco e palha de café in natura (Tabela 2). Esse comportamento corrobora as observações feitas por Guerrini e Trigueiro (2004), que constataram um aumento da densidade e do percentual de microporos com o acréscimo de lodo de esgoto na casca de arroz carbonizada.

Tabela 2. Caracterização física dos substratos formulados com diferentes proporções de resíduos orgânicos. Densidade aparente (Da), porosidade total (PT), espaço de aeração (EA), macroporosidade (Macrop), microporosidade (Microp), água facilmente disponível (AFD), água tamponante (AT) e água disponível (AD).

Table 2. Physical characterization of substrates formulated with different proportions of organic waste. Bulk density (Da), total porosity (TP), aeration space (EA), macroporosity (Macrop), microporosity (Microp), easily available water (AFD), buffering water (AT) and available water (AW).

\begin{tabular}{|c|c|c|c|c|c|c|c|c|}
\hline \multirow{2}{*}{ Tratamentos } & \multirow{2}{*}{$\begin{array}{c}\text { Da } \\
\text { g.cm } \\
\end{array}$} & PT & EA & Macrop & Microp & AFD & $\mathbf{A T}$ & AD \\
\hline & & \multicolumn{7}{|c|}{$(\%)$} \\
\hline T1 (100\% LE) & 0,21 & 75 & 17 & 23 & 52 & 25 & 5 & 30 \\
\hline $\mathrm{T} 2$ (80\% LE + 20\% FC) & 0,23 & 76 & 17 & 22 & 54 & 26 & 6 & 31 \\
\hline $\mathrm{T} 3$ (60\% LE + 40\% FC) & 0,17 & 72 & 12 & 20 & 52 & 28 & 6 & 34 \\
\hline $\mathrm{T} 4$ (40\% LE + 60\% FC) & 0,11 & 65 & 9 & 19 & 46 & 25 & 6 & 31 \\
\hline T5 (20\% LE + 80\% FC) & 0,07 & 47 & 9 & 17 & 30 & 15 & 3 & 18 \\
\hline T6 (80\% LE + 20\% PCN) & 0,18 & 75 & 24 & 28 & 47 & 18 & 2 & 21 \\
\hline $\mathrm{T} 7$ (60\% LE + 40\% PCN) & 0,17 & 73 & 31 & 26 & 47 & 11 & 2 & 14 \\
\hline $\mathrm{T} 8$ (40\% LE + 60\% PCN) & 0,10 & 73 & 35 & 29 & 44 & 7 & 1 & 8 \\
\hline T9 (20\% LE + 80\% PCN) & 0,05 & 72 & 38 & 31 & 41 & 2 & 0 & 2 \\
\hline T10 (100\% SC) & 0,32 & 85 & 26 & 33 & 52 & 19 & 3 & 23 \\
\hline
\end{tabular}

LE: lodo de esgoto; FC: fibra de coco; PCN: palha de café in natura; SC: substrato comercial à base de casca de pinus e vermiculita.

De acordo com a classificação de Gonçalves e Poggiani (1996), os tratamentos T1 (100\% LE), T2 (80\% LE + 20\% FC), T6 (80\% LE + 20\% PCN) e T10 (100\% SC) ofereceram porosidade total adequada, enquanto que no tratamento T5 (20\% LE + 80\% FC) ela foi considerada baixa e nos demais tratamentos, média. O lodo de esgoto puro (T1) possui porosidade total adequada, e a sua combinação em altas proporções com outros materiais, como na que ocorre com a fibra de coco, pode diminuir a porosidade total dos substratos, como ocorre nos tratamentos T5 (Tabela 2).

De acordo com Gonçalves e Poggiani (1996), os tratamentos T4 (40\% LE + 60\% FC) e T5 (20\% LE + 80\% FC) forneceram baixa macroporosidade, sendo que os demais tratamentos proporcionaram macroporosidade considerada média. Apesar dos valores baixos e médios de macroporosidade, Silva et al. (2012) ressaltam que esses valores sugeridos na literatura como adequados sejam utilizados com mais 
critério, uma vez que eles não se aplicam a todas as espécies, tipos de recipientes, formas de propagação, manejos hídricos e nutricionais e materiais utilizados na composição dos substratos. Já a microporosidade foi considerada adequada para a maioria dos tratamentos, sendo considerados médios apenas os tratamentos T5 (20\% LE + 80\% FC), T8 (40\% LE + 60\% PCN) e T9 (20\% LE + 80\% PCN), em que se observou uma diminuição da microporosidade quando foi incluído resíduo orgânico na composição do substrato. De forma geral, é necessário que haja um equilíbrio entre macro e microporos, uma vez que altas proporções de partículas maiores aumentam o espaço de aeração do substrato, fazendo com que ele não retenha água para disponibilizar às mudas, enquanto que partículas menores promovem o fechamento dos poros, aumentando a capacidade de retenção de água e diminuindo o espaço de aeração, o que prejudica o crescimento das raízes (FERMINO, 2003; FERRARI, 2003).

Os tratamentos T5 (20\% LE + 80\% FC), T7 (60\% LE + 40\% PCN), T8 (40\% LE + 60\% PCN) e T9 (20\% LE + 80\% PCN) apresentaram quantidade de água disponível (AD) abaixo do limite mínimo de 20\% proposto por Fermino (1996). Observou-se que, à medida que se acrescentavam maiores proporções de palha de café in natura, diminuía a quantidade de AD que foi também acompanhada pela diminuição não acentuada da microporosidade, a qual, por sua vez, pode ser responsável pela capacidade de retenção de água. Alguns tratamentos cuja microporosidade foi considerada adequada apresentaram valores de água disponível abaixo do limite mínimo considerado por Fermino (1996), como é o caso do tratamento T7 (60 LE + 40 PCN), que apresentou quantidade de água disponível entre 5 e 17\%.

O teor de água facilmente disponível (AFD) adequado para as plantas deve estar entre 75 e 90\% do valor total de água disponível, ou seja, devendo ser de 20 a 30\%, de acordo com De Boodt e Verdonck (1972). Dentro dessa faixa, encontram-se os tratamentos T1 (100\% LE), T2 (80\% LE + 20\% FC), T3 (60\% LE + 40\% FC) e T4 (40\% LE + 60\% FC). A utilização de materiais de baixa densidade e granulometria mais grosseira para a composição de substratos, como, por exemplo, a palha de café in natura, proporcionaram um maior espaço de aeração. Nesse caso, houve uma maior drenagem do substrato, que resultou em menores valores de AFD, concordando com Gonçalves e Poggiani (1996), que ressaltam que materiais com baixa densidade elevam a macroporosidade das misturas e reduzem a capacidade de retenção de água do substrato.

No geral, é possível observar que, à medida que aumenta a proporção de fibra de coco nos substratos, ocorre diminuição da densidade aparente, da porosidade total, da macroporosidade e da microporosidade. Aumentando-se a proporção de palha de café in natura, tem-se diminuição da densidade aparente e da água disponível.

\section{Características químicas dos substratos}

De forma geral, os valores de $\mathrm{pH}$ aumentaram à medida que se aumentaram as proporções de fibra de coco e palha de café in natura (Tabela 3). Esse efeito pode ser explicado pelo fato de esses resíduos oferecerem individualmente valores de $\mathrm{pH}$ dentro da faixa de 6,0 e 6,5. Caldeira et al. (2007) ressaltam que os componentes escolhidos para mistura dos substratos devem possuir $\mathrm{pH}$ dentro da faixa recomendada, para que a composição resultante mantenha-se dentro da faixa de tolerância.

$\mathrm{O}$ pH em água do substrato deve variar entre 6,0 e 6,5, de acordo com Rodrigues et al. (2002) e Fett (2005). Com exceção do tratamento T10 (100\% SC), todos os tratamentos apresentaram baixos valores de pH, o que, de acordo com Fett (2005), pode trazer problemas à formação da muda, devido à indisponibilidade de alguns nutrientes e fitotoxicidade, que pode ser desencadeada com a ocorrência de valores abaixo ou acima do limite considerado adequado. De forma geral, aumentando-se as proporções de fibra de coco e palha de café in natura, ocorre aumento do $\mathrm{pH}$, que pode ser explicado pelo fato de os materiais de composição dos substratos obterem pH em torno de 6,3 a 6,5 e uma diminuição nos teores totais de sais solúveis (TTSS) aos substratos.

Uma provável explicação para os altos valores de CTC verificados em praticamente todos os tratamentos do presente trabalho pode ser a matéria orgânica acrescentada aos substratos. Segundo Bezerra et al. (2006), a matéria orgânica pode alterar o complexo coloidal, criar cargas superficiais e, consequentemente, aumentar a CTC do solo (substrato), como observado com os substratos formulados com PCN, em que, à medida que se aumentava a dose de palha de café in natura, aumentava o valor de CTC, como também é descrito por Carneiro (1995), o qual afirma que o aumento da CTC está relacionado principalmente ao aumento do teor de matéria orgânica, mas que esse efeito varia de acordo com a fonte de matéria orgânica, em função da relação ácidos húmicos/ácidos fúlvicos. 
Tabela 3. Características químicas dos substratos formulados com diferentes proporções de resíduos orgânicos.

Table 3. Chemical characteristics of substrates formulated with different ratios of organic waste.

\begin{tabular}{|c|c|c|c|c|c|c|c|c|c|}
\hline \multirow{2}{*}{ Tratamentos } & \multirow{2}{*}{$\begin{array}{c}\mathbf{p H} \\
\mathbf{H}_{2} \mathrm{O} \\
\end{array}$} & $\mathbf{A l}$ & $\mathrm{H}+\mathrm{Al}$ & CTC & SB & $\mathbf{V}$ & $\mathbf{m}$ & \multirow{2}{*}{$\begin{array}{c}\mathrm{CE} \\
\mathrm{mS} . \mathrm{cm}^{-1}\end{array}$} & \multirow{2}{*}{$\begin{array}{l}\text { TTSS } \\
\text { g.L }{ }^{-1}\end{array}$} \\
\hline & & \multicolumn{4}{|c|}{$\mathrm{cmol}_{\mathrm{c}} \cdot \mathrm{dm}^{-3}$} & \multicolumn{2}{|c|}{$\%$} & & \\
\hline T1 (100\% LE) & 4,0 & 0,9 & 23,0 & 41,03 & 18,04 & 44,0 & 4,8 & 1,42 & 6,22 \\
\hline $\mathrm{T} 2$ (80\% LE + 20\% FC) & 4,0 & 1,0 & 18,5 & 36,23 & 17,74 & 49,0 & 5,3 & 0,89 & 2,76 \\
\hline T3 (60\% LE + 40\% FC) & 4,1 & 0,6 & 21,0 & 36,43 & 15,43 & 42,4 & 3,7 & 0,75 & 1,85 \\
\hline T4 (40\% LE + 60\% FC) & 4,4 & 0,4 & 24,2 & 35,09 & 10,93 & 31,2 & 3,5 & 0,66 & 1,20 \\
\hline T5 (20\% LE + 80\% FC) & 4,7 & 0,3 & 21,7 & 31,30 & 9,65 & 30,8 & 2,5 & 0,49 & 0,61 \\
\hline T6 (80\% LE + 20\% PCN) & 4,4 & 0,9 & 24,7 & 50,88 & 26,23 & 51,5 & 3,3 & 0,91 & 3,05 \\
\hline T7 (60\% LE + 40\% PCN) & 4,7 & 1,5 & 24,0 & 50,44 & 26,45 & 52,4 & 5,4 & 0,87 & 2,45 \\
\hline T8 (40\% LE + 60\% PCN) & 5,0 & 1,5 & 24,7 & 54,50 & 29,84 & 54,8 & 4,8 & 0,80 & 1,80 \\
\hline T9 (20\% LE + 80\% PCN) & 5,3 & 1,9 & 23,3 & 56,02 & 32,69 & 58,4 & 5,5 & 0,98 & 1,65 \\
\hline T10 (100\% SC) & 6,6 & 0,0 & 7,0 & 35,27 & 28,28 & 80,2 & 0,0 & 0,94 & 3,17 \\
\hline
\end{tabular}

LE: lodo de esgoto; FC: fibra de coco; PCN: palha de café in natura; SC: substrato comercial à base de casca de pinus e vermiculita.

Röber e Schaller (1985) estabelecem os seguintes limites de tolerância para a salinidade dos substratos: baixa $\left(<1,0\right.$ g.L $\left.{ }^{-1}\right)$, normal $\left(1,0\right.$ a 2,0 g.L $\left.{ }^{-1}\right)$, alta $\left(2,0\right.$ a 4,0 g.L $\left.{ }^{-1}\right)$, muito alta $\left(4,0\right.$ a 5,0 g.L $\left.L^{-1}\right)$, extremamente alta $\left(5,0\right.$ a 7,0 g. $\left.\mathrm{L}^{-1}\right)$ e tóxica $\left(>7,0\right.$ g.L $\left.\mathrm{L}^{-1}\right)$. Nesse sentido, são considerados limites ideais os tratamentos T3 (60\% LE + 40\% FC), T4 (40\% LE + 60\% FC), T8 (40\% LE + 60\% PCN) e T9 (20\% LE + $80 \%$ PCN). O tratamento T5 (20\% LE + 80\% FC) é considerado baixo. Para Fermino (2002), na interpretação do teor total de sais solúveis (TTSS), que expressa a concentração de sais em determinado volume de substrato, não se deve observar apenas a condutividade elétrica, mas considerar a densidade do material, pois, para o mesmo valor de condutividade elétrica, maior será a salinidade quanto maior for a densidade do material.

Com base na afirmação de Fermino (2002), T5 apresenta uma das menores médias de densidade e consequentemente menor valor de TTSS. O tratamento T1 (100\% LE) apresentou teor extremamente alto, segundo a referida classificação, o que pode estar relacionado com seu maior teor de condutividade elétrica. Os demais tratamentos apresentaram altos teores de sais solúveis. A salinidade é um dos itens a ser levado em consideração na escolha do material, buscando-se obter materiais com salinidade abaixo de 1,0 g.L ${ }^{-1}$ (KÄMPF, 2005). Neste caso, apenas T5 se enquadra nos limites propostos pelo autor.

De modo geral, verifica-se aumento da salinidade (TTSS) à medida que são adicionadas maiores proporções de lodo de esgoto à composição do substrato. A salinidade dos substratos durante o cultivo das mudas possivelmente atingiu valores ainda maiores dos que os apresentados na análise química, que foi realizada com amostras sem a adição da adubação de base.

A lixiviação dos sais devido às irrigações constantes ou mesmo a tolerância da espécie utilizada a altos níveis de salinidade no substrato podem ser prováveis explicações para os resultados obtidos, conforme também observado por Kratz (2011) em Eucalyptus benthamii Maiden et Cambage.

De acordo com Gonçalves et al. (2000), a condutividade elétrica do substrato não deve exceder o limite de $1,0 \mathrm{mS} . \mathrm{cm}^{-1}$. Nesse sentido, todos os tratamentos apresentaram valores de condutividade elétrica dentro da faixa considerada adequada, com exceção do tratamento T1 (100\% LE), que, por possuir altos teores de nutrientes, teve aumento da sua condutividade elétrica. A condutividade é afetada pela quantidade de fibra de coco no substrato, indicando que, quanto maior a proporção desse material ou menor a proporção de lodo de esgoto, menor a condutividade elétrica do substrato, também observado por Guerrini e Trigueiro (2004), em que o aumento da concentração de biossólido provocou aumento na condutividade elétrica, visto a alta concentração de sais desse material.

Com relação à soma de bases, a fibra de coco e a palha de café in natura tiveram influência diferente no comportamento desse parâmetro. À medida que se aumentou a proporção de fibra de coco, diminuiu a soma de bases, o que pode ser explicado pelo fato de diminuição dos valores das bases, com exceção do $\mathrm{K}$, que aumentou com as doses de fibra de coco. O motivo principal da elevação do $\mathrm{K}$ é a constituição do material. Aumentando a proporção de palha de café in natura, aumentou-se a soma de bases do substrato, o que pode ser explicado pelo expressivo aumento do teor de $\mathrm{K}$ à medida que se 
aumentava a quantidade de PCN, fato que pode ser consolidado pela alta concentração dessa base no material constituinte dos substratos.

De acordo com a classificação de Prezotti et al. (2007), os teores trocáveis de K descritos na Tabela 4 são considerados médio para T1 (100\% LE) e alto para os demais tratamentos. Os tratamentos que continham palha de café in natura em sua composição, ou seja, os tratamentos T6 (80\% LE + 20\% PCN), T7 (60\% LE + 40\% PCN), T8 (40\% LE + 60\% PCN) e T9 (20\% LE + 80\% PCN), disponibilizaram altos teores de $\mathrm{K}$, segundo os limites propostos pelos mesmos autores, sendo que, à medida que se aumentavam as proporções desse resíduo, aumentavam também os teores de K. Marschner (1995) ressalta que o suprimento adequado de K é importante, por contribuir com o crescimento da planta, como resultado do equilíbrio entre N, P e K, além de reduzir a absorção excessiva de Mg.

Objetivando a caracterização química de diversos resíduos orgânicos, Silva et al. (2009) verificaram que a palha de café, dentre os resíduos vegetais testados, destacou-se em relação ao teor de K, que foi superior ao de todos os resíduos orgânicos avaliados. No referido estudo, a palha de café também resultou em grande interferência em relação ao K.

Quanto aos teores disponíveis de $\mathrm{Ca}$, de acordo com a recomendação proposta por Prezotti (2007) (Tabela 4), o tratamento T9 (20\% LE + 80\% PCN) é considerado médio, enquanto os demais disponibilizaram altos teores desse cátion. Verificou-se que nos tratamentos que continham fibra de coco e palha de café in natura os teores de Ca se reduziram à medida que se diminuiu a proporção de lodo de esgoto, ou seja, a utilização desses resíduos vegetais proporcionou redução dos teores disponíveis de Ca. Isso se deve ao fato de que esses materiais apresentam individualmente baixos teores de Ca, com exceção da palha de café in natura, e que, pelo fato de o lodo de esgoto possuir grandes quantidades de matéria orgânica, também disponibiliza maiores quantidades de Ca para o substrato.

Tabela 4. Teores disponíveis de macronutrientes dos substratos formulados com diferentes proporções de resíduos orgânicos.

Table 4. Available concentration of macronutrient substrates formulated with different proportions of organic waste.

\begin{tabular}{lcccc}
\hline \multirow{2}{*}{ Tratamentos } & $\mathbf{P}$ & $\mathbf{K}$ & $\mathbf{C a}$ & $\mathbf{M g}$ \\
\cline { 2 - 5 } & & $\mathbf{c m o l}_{\mathbf{c} . \mathbf{d m}} \mathbf{- 3}$ & & \\
\hline T1 (100\% LE) & 204 & 144 & 12,5 & 4,8 \\
T2 (80\% LE + 20\% FC) & 176 & 247 & 12,3 & 4,6 \\
T3 (60\% LE + 40\% FC) & 167 & 526 & 10,1 & 3,8 \\
T4 (40\% LE + 60\% FC) & 137 & 699 & 6,1 & 2,9 \\
T5 (20\% LE + 80\% FC) & 145 & 1105 & 4,7 & 2,0 \\
T6 (80\% LE + 20\% PCN) & 198 & 3230 & 14,2 & 3,6 \\
T7 (60\% LE + 40\% PCN) & 217 & 5640 & 8,7 & 3,2 \\
T8 (40\% LE + 60\% PCN) & 257 & 7860 & 6,2 & 3,3 \\
T9 (20\% LE + 80\% PCN) & 291 & 10160 & 3,4 & 3,2 \\
T10 (100\% SC) & 189 & 470 & 13,1 & 13,9 \\
\hline
\end{tabular}

É possível observar que, quanto maior a proporção de fibra de coco e palha de café in natura, maior a disponibilidade dos teores de K. No entanto, o inverso ocorreu para os teores de Ca e Mg, indicando baixa disponibilidade desses materiais e sugerindo que, quanto maior a proporção de lodo de esgoto no substrato, maior será a disponibilidade de Ca e Mg. Também é possível observar que maiores proporções de palha de café in natura contribuíram para aumento da disponibilidade de P do substrato.

\section{Características morfológicas}

A análise de variância dos tratamentos compostos por resíduos revelou diferenças significativas, em nível de 5\% de probabilidade, para altura da parte aérea (H), diâmetro do coleto (D), massa seca da parte aérea (MSPA), massa seca radicular (MSR) e massa seca total (MST). Na tabela 5 estão apresentados os valores médios dos tratamentos para os parâmetros acima relacionados, comparados pelo teste de Scott-Knott.

Os maiores crescimentos em altura foram observados nos tratamentos T1 (100\% LE), T2 (80\% $\mathrm{LE}+20 \% \mathrm{FC})$, T3 (60\% LE + 40\% FC), T6 (80\% LE + 20\% PCN) e T8 (40\% LE + 60\% PCN), demonstrando que maiores proporções de lodo de esgoto parecem ser favoráveis ao crescimento de 
mudas de Eucalyptus grandis. A altura mínima indicada para o plantio, segundo Wendling e Dutra (2010), é de 15 cm. Nesse sentido, apenas os tratamentos T5 (20\% LE + 80\% FC) e T10 (100\% SC) não proporcionaram mudas com altura adequada ao plantio em campo. Os tratamentos T4 (40\% LE + $60 \%$ FC), T5 (20\% LE + 80\% FC) e T10 (100\% SC) apresentaram crescimento estatisticamente inferior aos demais, demonstrando que a utilização de substrato comercial puro ou em proporções acima de $60 \%$ de fibra de coco na composição do substrato promovem um desenvolvimento aquém do desejado. Utilizando lodo de esgoto e casca de arroz carbonizada para a produção de mudas de Eucaliptus grandis, Rocha et al. (2013) verificaram que o substrato com 100\% de lodo de esgoto proporcionou a maior altura para as mudas. No presente estudo, as maiores proporções de lodo de esgoto foram responsáveis por maiores médias de altura. Por meio dos resultados da análise química dos teores de macronutrientes (Tabela 4), foi possível observar a diminuição dos teores de P, Ca e Mg dos substratos com a diminuição do lodo de esgoto, o que pode estar associado às maiores médias da altura das mudas com maiores proporções do lodo de esgoto.

Tabela 5. Altura da parte aérea (H), diâmetro do coleto (D), massa seca da parte aérea (MSPA), massa seca radicular (MSR) e massa seca total (MST) de mudas de Eucalyptus grandis produzidas com diferentes proporções de resíduos orgânicos.

Table 5. Shoot height (H), diameter (D), dry matter (SDM), root dry mass (RDM) and total dry matter (TDM) of Eucalyptus grandis seedlings produced with different proportions of organic waste.

\begin{tabular}{|c|c|c|c|c|c|}
\hline \multirow{2}{*}{ Tratamentos } & \multirow{2}{*}{$\begin{array}{c}\mathbf{H} \\
\mathbf{c m}\end{array}$} & \multirow{2}{*}{$\underset{\mathbf{m m}}{\mathrm{D}}$} & MSPA & MSR & MST \\
\hline & & & \multicolumn{3}{|c|}{ g.planta ${ }^{-1}$} \\
\hline T1 (100\%LE) & $26,12 a^{1}$ & $2,58 \mathrm{~b}$ & $0,941 \mathrm{c}$ & $0,262 \mathrm{c}$ & $1,203 \mathrm{c}$ \\
\hline $\mathrm{T} 2$ (80\%LE+20\%FC) & 24,79 a & $2,51 \mathrm{~b}$ & $0,749 \mathrm{c}$ & $0,194 \mathrm{c}$ & $0,944 \mathrm{~d}$ \\
\hline T3 (60\%LE 40\%FC) & 26,35 a & $2,66 \mathrm{~b}$ & $0,836 \mathrm{c}$ & $0,174 \mathrm{c}$ & $1,011 \mathrm{~d}$ \\
\hline T4 (40\%LE+60\%FC) & 16,65 c & $1,86 \mathrm{c}$ & $0,364 \mathrm{~d}$ & $0,141 \mathrm{c}$ & $0,506 \mathrm{e}$ \\
\hline T5 (20\%LE+80\%FC) & $13,40 \mathrm{c}$ & $1,51 \mathrm{~d}$ & $0,553 \mathrm{~d}$ & $0,309 \mathrm{c}$ & $0,862 \mathrm{~d}$ \\
\hline T6 (80\%LE+20\%PCN) & 30,84 a & 3,07 a & $1,891 \mathrm{a}$ & $0,844 \mathrm{a}$ & $2,736 \mathrm{a}$ \\
\hline T7 (60\%LE+40\%PCN) & $19,99 \mathrm{~b}$ & $2,25 \mathrm{~b}$ & $1,019 \mathrm{c}$ & $0,444 \mathrm{~b}$ & $1,463 \mathrm{c}$ \\
\hline T8 (40\%LE+60\%PCN) & 27,40 a & $2,86 \mathrm{a}$ & $1,291 \mathrm{~b}$ & $0,538 \mathrm{~b}$ & $1,829 \mathrm{~b}$ \\
\hline T9 (20\%LE+80\%PCN) & $21,93 \mathrm{~b}$ & $2,48 \mathrm{~b}$ & 0,992 c & $0,427 \mathrm{~b}$ & $1,420 \mathrm{c}$ \\
\hline T10 (100\%SC) & $9,85 \mathrm{c}$ & $1,28 \mathrm{~d}$ & $0,143 \mathrm{~d}$ & $0,021 \mathrm{~d}$ & $0,165 \mathrm{e}$ \\
\hline
\end{tabular}

LE: lodo de esgoto; FC: fibra de coco; PCN: palha de café in natura; SC: substrato comercial à base de casca de pinus e vermiculita.

${ }^{1}$ Médias seguidas de uma mesma letra não diferem entre si pelo teste Scott-Knott, ao nível de 5\% de probabilidade.

Características intrínsecas dos materiais utilizados na composição dos substratos podem influenciar o desenvolvimento radicular e consequentemente o crescimento da parte aérea, como é o caso do tratamento T5 (20\% LE + 80\% FC), que apresentou baixos valores de água disponível (AD) e baixa macroporosidade (macro), conforme análise física realizada no presente trabalho.

Para o crescimento em diâmetro do coleto, os tratamentos T6 (80\% LE + 20\% PCN) e T8 (40\% $\mathrm{LE}+60 \% \mathrm{PCN}$ ) foram considerados estatisticamente superiores e encontram-se aptos para o plantio, segundo Wendling e Dutra (2010), que consideram como adequado um diâmetro do coleto acima de $2 \mathrm{~mm}$ para mudas de eucalipto. Tomando-se por base esse quesito, só não podem ser consideradas aptas as mudas crescidas nos tratamentos T4 (40\% LE + 60\% FC), T5 (20\% LE + 80\% FC) e T10 (100\% SC), que proporcionaram médias estatísticas inferiores. Semelhante ao presente estudo, Rocha et al. (2013) verificaram que o substrato comercial não alcançou as dimensões recomendadas. Foi possível observar que os tratamentos com os menores valores médios para o diâmetro do coleto, ou seja, com 60 e $80 \%$ de fibra de coco, proporcionaram às mudas as menores médias dos teores de $\mathrm{P}$, Ca e Mg, mostrando a importância dos mesmos ao crescimento em diâmetro das mudas.

Apenas o tratamento T6 (80\% LE + 20\% PCN) pode ser considerado estatisticamente superior aos demais, no quesito massa seca da parte aérea, enquanto que os tratamentos T4 (40\% LE + 60\% FC), T5 (20\% LE + 80\% FC) e T10 (100\% SC) proporcionaram médias inferiores, conforme o esperado, visto que esses tratamentos também proporcionaram crescimento em altura e diâmetro inferiores.

Os resultados do presente estudo revelaram que o plantio de mudas de Eucalyptus grandis em substrato comercial composto por casca de pinus decomposta e vermiculita, ou em substratos que 
contenham proporções de fibra de coco maiores que $60 \%$ na sua composição, mostrou-se desfavorável ao crescimento da parte aérea das mudas, quando comparado aos demais tratamentos testados. Essa observação pode estar relacionada ao fato de que não houve fertilizações de cobertura no substrato comercial, não proporcionando assim condições para o bom crescimento das mudas, ainda que seus atributos físicos sejam considerados adequados. Discordando desses resultados, Trigueiro e Guerrini (2003) verificaram que a produção de massa seca da parte aérea em mudas de Eucalyptus grandis aos 120 dias foi superior com a utilização de substrato comercial (à base de casca de pinus, vermiculita, húmus e terra vegetal), em relação aos demais tratamentos que continham diferentes proporções de lodo de esgoto e casca de arroz carbonizada em sua composição.

Para a massa seca radicular, o tratamento T6 (80\% LE + 20\% PCN) foi considerado estatisticamente superior aos demais, conforme ocorrido também para as características altura, diâmetro e massa seca da parte aérea. O tratamento T6 (80\% LE + 20\% PCN) foi o que proporcionou a maior média para os teores de Ca disponível para o substrato. O Ca proporciona um maior condicionamento do substrato e maior exploração das raízes. O substrato comercial apresentou média elevada para esse nutriente, no entanto, proporcionou médias inferiores ao tratamento T6 em relação a P e K.

Acompanhando o desenvolvimento estatisticamente superior em termos de massa seca da parte aérea e massa seca radicular, o tratamento T6 (80\% LE + 20\% PCN) também proporcionou médias de massa seca total superiores à dos demais tratamentos, visto que essa característica é o somatório das anteriormente citadas. Dessa forma, a combinação entre lodo de esgoto e palha de café in natura, nas proporções de 80 e $20 \%$, respectivamente, parece ser favorável ao crescimento das mudas de Eucalyptus grandis como um todo.

\section{CONCLUSÕES}

- As diferentes proporções dos resíduos e lodo de esgoto promoveram diferentes resultados das características químicas e físicas dos substratos formulados. Nenhum substrato testado apresentou valores considerados ideais em todos os atributos estudados.

- Mudas produzidas com $80 \%$ de lodo de esgoto + 20\% de palha de café in natura apresentam maiores crescimento em altura, diâmetro do coleto, massa seca da parte aérea, massa seca radicular e massa seca total, sendo essa composição a mais indicada para a produção de mudas entre as demais testadas no presente estudo.

- Considerando que não houve fertilizações de cobertura, o substrato comercial não proporcionou condições para o bom crescimento das mudas, porém seus atributos físicos são considerados adequados.

\section{REFERÊNCIAS}

BEZERRA, F. B.; OLIVEIRA, M. A. C. L.; PEREZ, D. V.; ANDRADE, A. G.; MENEGUELLI, N. A. Lodo de esgoto em revegetação de área degradada. Pesquisa Agropecuária Brasileira, Brasília: v. 41, n. 3. p. 469 - 476, 2006.

BRASIL. Ministério do Meio Ambiente, Conselho Nacional de Meio Ambiente, CONAMA. Resolução CONAMA no 375/2006, de 30 de agosto de 2006 - In: Resoluções, 2006. Disponível em: $<$ http://www.mma.gov.br>. Acesso em: 20/09/2011.

CALDEIRA, M. V. W.; MARCOLIN, M.; MORAES, E.; SCHAADT, S. S. Influência do resíduo da indústria do algodão na formulação de substrato para produção de mudas de Schinus terebinthifolius Raddi, Archontophoenix alexandrae Wendl. et Drude e Archontophoenix cunninghamiana Wendl. et Drude. Ambiência, Guarapuava, v. 3, p. 1 - 8, 2007.

CARNEIRO, J. G. A. Produção e controle de qualidade de mudas florestais. Curitiba: UFPR/FUPEF, 1995. $451 \mathrm{p}$.

DE BOODT, M.; VERDONCK, O. The physical properties of the substrates. In: DE BOODT, M.; VERDONCK, O. The physical properties of the substrates in horticulture. Acta Horticulturae, Wageningen: n. 26, p. 37 - 44, 1972. 
FERMINO, M. H. Aproveitamento de resíduos industriais e agrícolas como alternativas de substratos hortícolas. 90 f. Dissertação (Mestrado em Fitotecnia) - Universidade Federal do Rio Grande do Sul, Porto Alegre, 1996.

Métodos de análise para caracterização física de substratos. 89 f. Tese (Doutorado em Fitotecnia) - Universidade Federal do Rio Grande do Sul, Porto Alegre, 2003.

FERMINO, M. H. O. Uso da análise física na avaliação da qualidade de componentes e substratos. In: FURLANI, A. M. C. et al. Caracterização, manejo e qualidade de substratos para a produção de plantas. Campinas: Instituto Agronômico, 2002. p. 29 - 37.

FERRARI, M. P. Cultivo do Eucalipto: produção de mudas. sistemas de produção 4. Versão Eletrônica. 2003. Disponível em:<http://sistemasdeproducao.cnptia.embrapa.br/FontesHTML/Eucalipto/Cultivodo Eucalipto/03_producao_de_mudas.htm>. Acesso em: 30/10/2013.

FETT, M. S. Serviço Brasileiro de Respostas Técnicas. Agricultura e pecuária. SENAI/Rio Grande do Sul/Departamento Regional. 2005. Disponível em:<http://www.sbrt.ibict.br>. Acesso em: 11/12/2011.

GONÇALVES, J. L. M.; POGGIANI, F. Substratos para produção de mudas florestais. In: CONGRESSO LATINO AMERICANO DE CIÊNCIA DO SOLO, 13., 1996, Águas de Lindoia, SP. Anais..., Águas de Lindoia, SP: USP- ESALQ/SBCS/CEA/SLACS/SBM, 1996. CD-ROM.

GONÇALVES, J. L. M.; SANTERELLI, E. G.; NETO, S. P. M.; MANARA, M. P. Produção de mudas de espécies nativas: substrato, nutrição, sombreamento e fertilização. In: GONÇALVES, J. L. M.; BENEDETTI, V. (eds.). Nutrição e fertilização florestal. Piracicaba: IPEF, 2000, p. 309 - 350.

GUERRINI, I. A.; TRIGUEIRO, R. M. Atributos físicos e químicos de substratos compostos por biossólidos e casca de arroz carbonizada. Revista Brasileira de Ciência do Solo, Viçosa, v. 28, p. 1069 1076, 2004.

JORGE, J. A.; CAMARGO, O. A.; VALADARES, J. M. A. S. Condições físicas de um Latossolo Vermelho-escuro quatro anos após aplicação de lodo de esgoto e calcário. Revista Brasileira de Ciências do Solo, Viçosa, v. 15, p. 237 - 240, 1991.

KÄMPF, A. N. Substrato. In: KAMPF, A. N. Produção comercial de plantas ornamentais. 2. ed. Guaíba: Agrolivros, 2005. p. 45 - 72.

KRATZ, D. Substratos renováveis para produção de mudas de Eucalyptus benthamii Maiden et cambage e Mimosa scabrella Benth. 118 f. Dissertação (Engenharia Florestal) - Universidade Federal do Paraná, Curitiba, 2011.

MARSCHNER, H. Mineral nutrition of higher plants. London: Academic Press, 1995. 889 p.

MATOS, A. T.; VIDIGAL S. M.; SEDIYAMA, M. A. N.; GARCIA, N. C. P.; RIBEIRO, M. F. Compostagem de alguns resíduos orgânicos utilizando-se águas residuárias da suinocultura como fonte de nitrogênio. Revista Brasileira de Engenharia Agrícola e Ambiental, Campina Grande, v. 2, n. 2, p. 199 - 203, 1998.

MELLO, R. P. Consumo de água do lírio asiático em vaso com diferentes substratos. 74 f. Dissertação (Mestrado em Engenharia Agrícola) - Universidade Federal de Santa Maria, Santa Maria, 2006.

MINISTÉRIO DA AGRICULTURA, PECUÁRIA E ABASTECIMENTO (MAPA). Instrução Normativa SDA no 17. Diário Oficial da União - Seção 1, nº 99, 24 de maio de 2007. Métodos Analíticos Oficiais para Análise de Substratos para Plantas e Condicionadores de Solo. Brasília, 2007.

MORAES NETO, S. P.; ABREU JR., C. H.; MURAOKA, T. Uso de biossólido em plantios florestais. Brasília: Embrapa Informações Tecnológicas; Embrapa Cerrados, Planaltina, 2007. v. 202, p. 1 - 16.

NEVES, J. M. G.; SILVA, H. P.; DUARTE, R. F. Uso de substratos alternativos para produção de mudas de moringas. Revista Verde de Agroecologia e Desenvolvimento Sustentável, Mossoró, v. 5, n. 1, p. 173 - 177, 2010. 
NUNES, M. U. C. Produção de mudas de hortaliças com o uso da plasticultura e do pó da casca de coco. Brasília: Embrapa Informações Tecnológicas; Embrapa Tabuleiros Costeiros, Aracaju, 2000. 29 p.

PREZOTTI, L. C.; GOMES, J. A.; DADALTO, G. G.; OLIVEIRA, J. A. Manual de recomendação de calagem e adubação para o Estado do Espírito Santo - $\mathbf{5}^{\mathbf{a}}$ aproximação. Vitória: SEEA/INCAPER/CEDAGRO, 2007. 305 p.

RÖBER, R.; SCHALLER, K. Pflanzenernährung im Gartenbau. Stuttgart, 1985. 352 p.

ROCHA, J. H. T.; BACKES, C.; DIOGO, F. A.; PASCOTTO, C. B.; BORELLI, K. Composto de lodo de esgoto como substrato para mudas de eucalipto. Pesquisa Florestal Brasileira, Colombo, v. 33, p. 27 35, 2013.

RODRIGUES, C. A. G.; BEZERRA, B. C.; ISHII, I. H.; CARDOSO, E. L.; SORIANO, B. M. A.; OLIVEIRA, H. O. Arborização urbana e produção de mudas de essências florestais nativas em Corumbá, MS. Brasília: Embrapa Informações Tecnológicas; Corumbá: Embrapa Pantanal, 2002. 26 p.

SILVA, C. A.; HIGASHIKAWA, F. S.; BETTIOL, W. Caracterização química de resíduos visando a avaliação de valor agronômico. In: CONGRESSO BRASILEIRO DE RESÍDUOS ORGÂNICOS, 2009, Vitória, ES. Anais... Vitória: CBRO, 2009. 1 CD-ROM.

SILVA, F. C. da. (org.). Manual de análises químicas de solos, plantas e fertilizantes. Brasília: Embrapa Informações Tecnológicas, 2, ed. 2009. 627 p.

SILVA, R. B. G. da; SIMÕES, D.; SILVA, M. R. Qualidade de mudas clonais de Eucalyptus urophylla x E. grandis em função do substrato. Revista Brasileira de Engenharia Agrícola e Ambiental, Campina Grande, v. 16, p. 297 - 302, 2012.

TRIGUEIRO, R. de M.; GUERRINI, I. A. Uso de biossólido como substrato para produção de mudas de eucalipto. Scientia Forestalis, Piracicaba, v. 64, p. 150 - 162, 2003.

VEGRO, C. L.; CARVALHO, F. C. de. Disponibilidade e utilização de resíduos no processamento agroindustrial do café. Informações Econômicas, São Paulo, v. 24, n. 1, p. 9 - 16, 1994.

WENDLING, I.; DUTRA, L. F. Produção de mudas de eucalipto por sementes. In: WENDLING, I.; DUTRA, L. F. Produção de mudas de eucalipto. Brasília: Embrapa Informações Tecnológicas; Embrapa Florestas, Colombo, 2010. p. 13 - 47.

WENDLING, I.; GATTO, A. Substratos, adubação e irrigação na produção de mudas. Viçosa, 2002. $146 \mathrm{p}$. 
